\title{
Two Cases of Restrictive Cardiomyopathy in Children
}

\author{
Mitsuhiro Kamisago, Takashi Ohkubo ${ }^{1,2}$, Makoto Watanabe', \\ Ei Ikegami ${ }^{1}$, Ryuji Fukazawa ${ }^{1}$ and Shunichi Ogawa ${ }^{1}$ \\ ${ }^{1}$ Department of Pediatrics, Graduate School of Medicine, Nippon Medical School \\ ${ }^{2}$ Department of Pediatrics, Saitama Red Cross Hospital
}

\begin{abstract}
A 3-year-old girl was diagnosed with restrictive cardiomyopathy (RCM) after showing symptoms of heart failure, and a 6-year-old boy was found to have RCM after abnormal electrocardiographic findings were seen during school-based heart disease screening. Both had typical clinical features of the disease. Plasma levels of brain natriuretic peptide increased significantly in both patients, allowing us to distinguish this disease from constrictive pericarditis which has similar clinical and hemodynamic features. The early diastolic mitral annular velocity recorded by tissue Doppler echocardiography was also useful to discriminate $\mathrm{RCM}$ from constrictive pericarditis. The former case successfully received heart transplantation, but the latter case died suddenly prior to receiving a heart transplant. The plasma level of brain natriuretic peptide and tissue Doppler echocardiography helped us to diagnose this disease earlier and follow it more carefully, which has important implications in optimal treatment and improved prognosis of RCM in children.
\end{abstract}

(J Nippon Med Sch 2009; 76: 313-318)

Key words: restrictive cardiomyopathy, brain natriuretic peptide, tissue Doppler echocardiography

\section{Introduction}

Restrictive cardiomyopathy (RCM) is a primary heart-muscle disease characterized by impaired ventricular filling with normal or decreased diastolic ventricular volume. Left ventricular systolic function usually remains normal, at least in the early phase of the disease, and the thickness of the left ventricle wall is within the normal range. It is a rare form of cardiomyopathy in childhood, accounting for between $2-5 \%$ of idiopathic cardiomyopathy in that age group ${ }^{1.2}$. It is often difficult to distinguish RCM from constrictive pericarditis, because of similar clinical features and similar ventricular diastolic filling hemodynamics. RCM may progress rapidly in childhood $^{3.4}$ and heart transplantation is the only reliable treatment in such cases. We report two cases of RCM, both with rapid progression to heart failure. The plasma level of brain natriuretic peptide (BNP) and the early diastolic mitral annular velocity (e') recorded by tissue Doppler echocardiography were found to be useful for diagnosis and follow-up of RCM.

\section{Case 1}

A 3-year-old girl was referred to our hospital with

Correspondence to Mitsuhiro Kamisago, Department of Pediatrics, Nippon Medical School Musashi Kosugi Hospital, 1-396 Kosugi-cho, Nakahara-ku, Kawasaki, Kanagawa 211-8533, Japan

E-mail: kamisago@nms.ac.jp

Journal Website (http://www.nms.ac.jp/jnms/) 


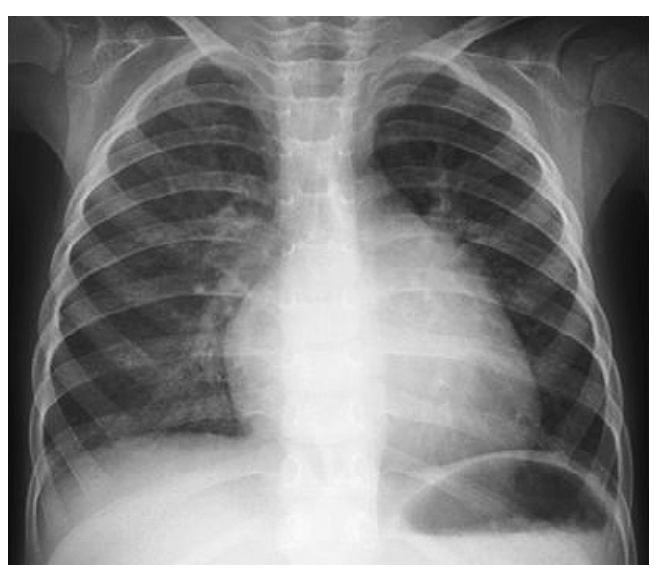

Fig. 1A Chest radiograph shows a cardiothoracic ratio of $56 \%$, with a double right cardiac silhouette produced by a markedly enlarged left atrium.
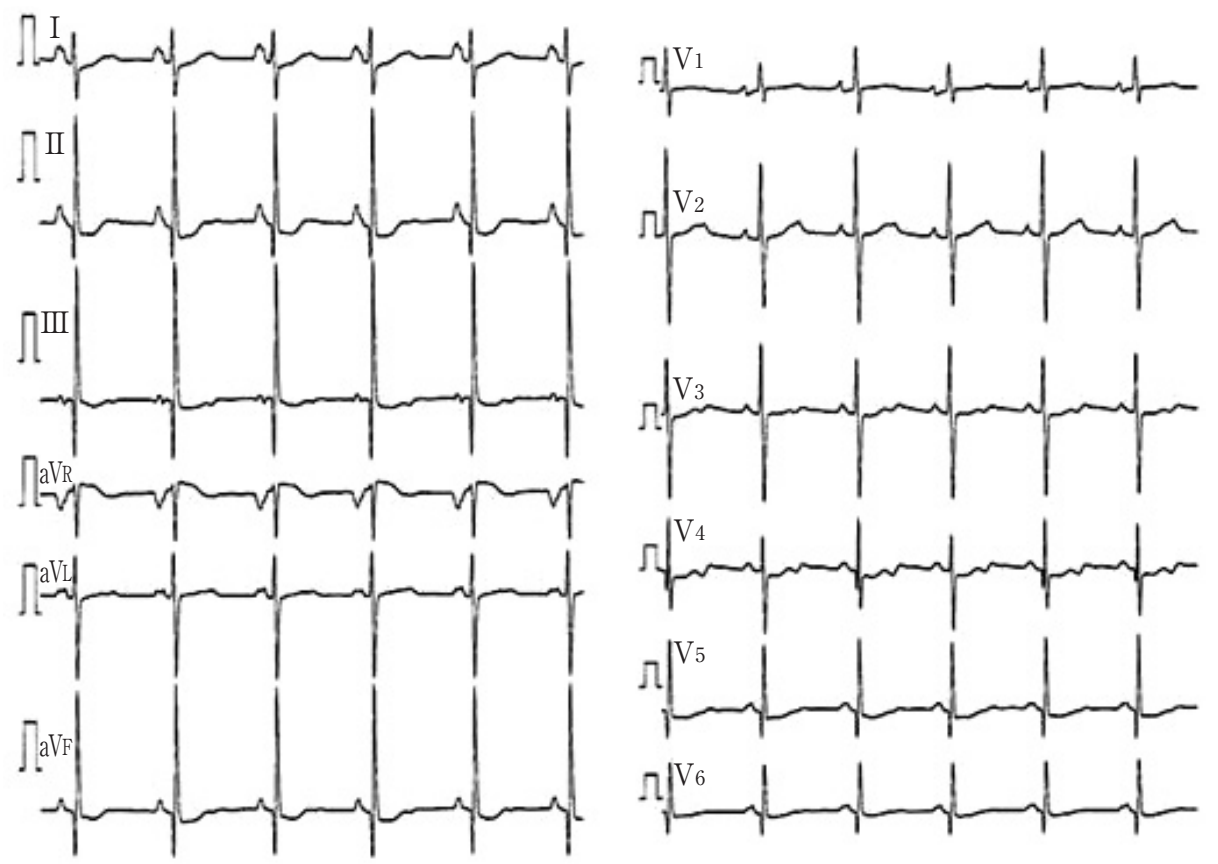

Fig. 1B Electrocardiogram shows left and right atrial enlargement, ST-segment depression, and low-voltage $\mathrm{T}$ waves in the inferior and lateral leads.

symptoms of cough, dyspnea on exertion, and hepatomegaly. At the age of 2 years 8 months, a bout of pneumonia led to chest radiography, which revealed cardiomegaly. Echocardiography suggested the diagnosis of RCM. At the time of referral, she was being treated with diuretics, $\beta$-blocking agents, angiotensin-converting enzyme inhibitors, isosorbide dinitrate, and aspirin. On physical examination, height was $92.8 \mathrm{~cm}$, weight was $12.2 \mathrm{~kg}$, systolic blood pressure was $92 \mathrm{~mm} \mathrm{Hg}$, and pulse was 78 beats per minute. No abnormal heart sounds were detected, and no significant heart murmur was audible. The liver edge was palpable 3 to $4 \mathrm{~cm}$ below the right costal margin, and the spleen was palpable $2 \mathrm{~cm}$ below the left costal margin. The plasma level of BNP was markedly elevated to 1,290 $\mathrm{pg} / \mathrm{mL}$. Chest radiography showed cardiomegaly (cardiothoracic ratio, 56\%), a double silhouette along the right cardiac border indicating left atrial enlargement, and mild pulmonary congestion (Fig. 1 

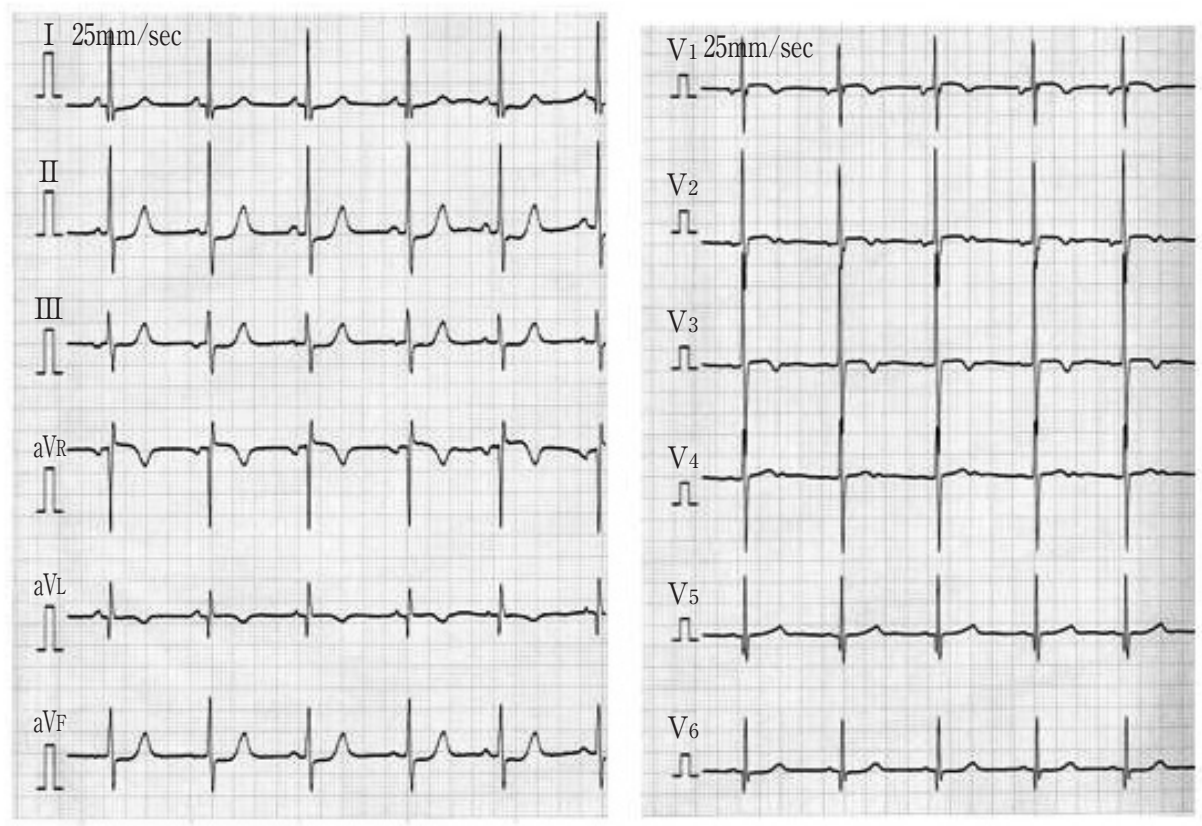

EKG (6ylm)

Fig. 2A Electrocardiogram shows left atrial enlargement, deep $\mathrm{Q}$ and low-voltage $\mathrm{T}$ waves in leads V5 and V6 and a 2.0-mV R wave in lead V1.

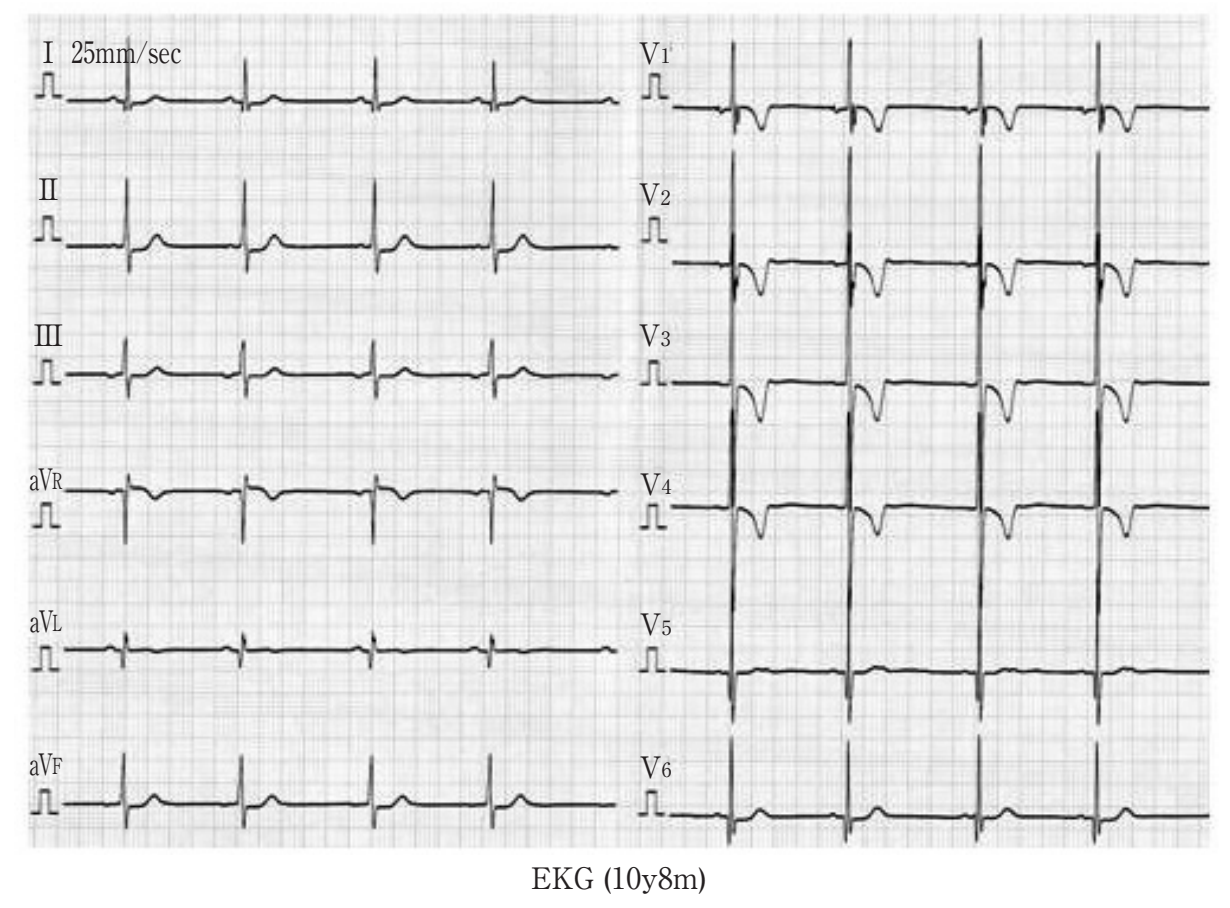

Fig. 2B Electrocardiogram shows left atrial enlargement and left- and right-ventricular hypertrophy. It also shows negative $\mathrm{T}$ waves in leads $\mathrm{V} 1$ to $\mathrm{V} 4$, low-voltage $\mathrm{T}$ waves in leads V5 and V6, ST-T depression in leads II and V6, and deep Q waves in leads V5 and V6.

A). Electrocardiography revealed sinus rhythm, left and right atrial enlargement, ST-segment depression, and low-voltage $\mathrm{T}$ waves in inferior and lateral leads (Fig. 1B). Echocardiography showed a anatomically normal heart with marked left atrial dilatation, a left atrial/aortic ratio of 3.48, mild mitral valve regurgitation, no tricuspid valve regurgitation, and mild left ventricular dysfunction with an 
$(\mathrm{pg} / \mathrm{mL})$

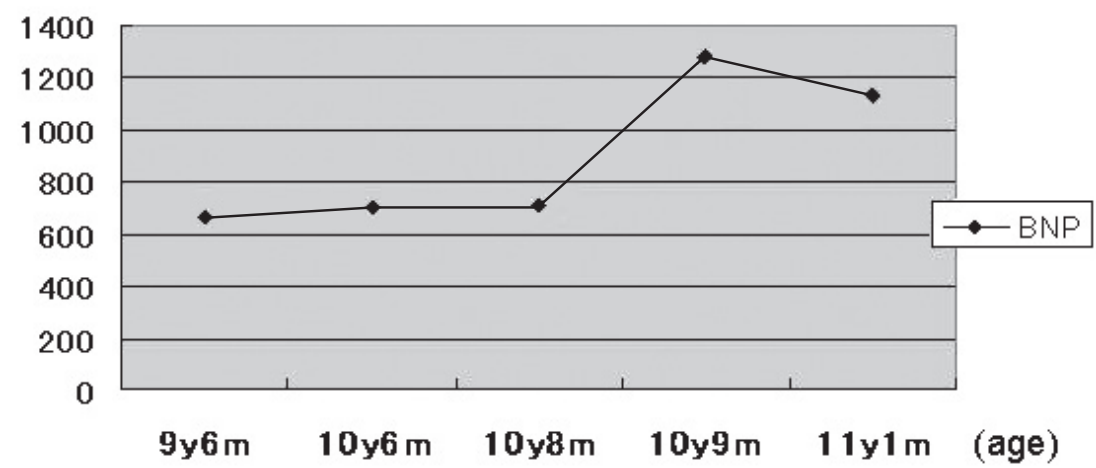

Fig. 3 This figure shows the time course of plasma levels of BNP in case 2. Elevated BNP indicates a clinically critical condition.

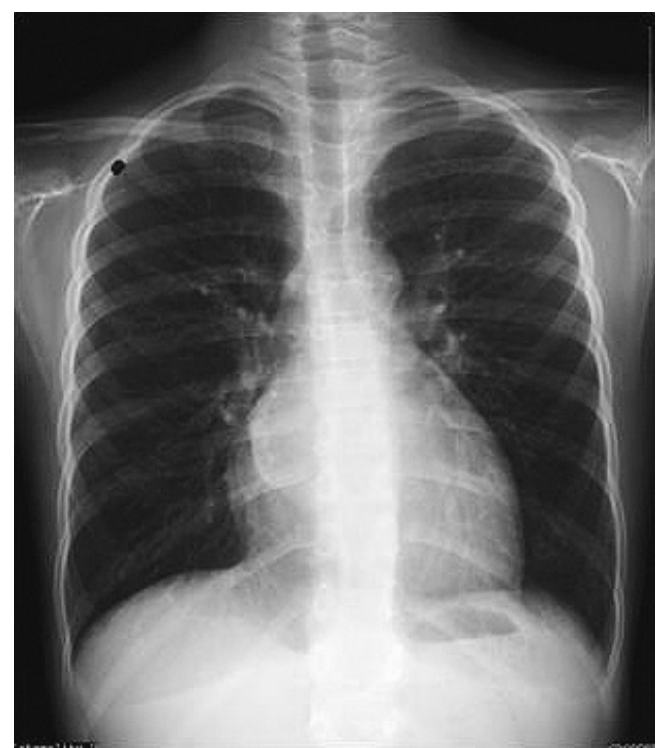

Fig. 4 Chest radiograph shows cardiomegaly with a cardiothoracic ratio of $54 \%$ and double right cardiac silhouette produced by a markedly enlarged left atrium.

ejection fraction of $56 \%$. The left ventricular enddiastolic dimension was $39.6 \mathrm{~mm}$ (reference range: 24-42 $\mathrm{mm}$ ), and the left ventricular end-diastolic wall thickness was $4.4 \mathrm{~mm}$ (reference range: $5^{-7} \mathrm{~mm}$ ). The patient also underwent cardiac catheterization. Hemodynamic measurements revealed an elevated end-diastolic pressure of $28 \mathrm{~mm} \mathrm{Hg}$ in the left ventricle and $10 \mathrm{~mm} \mathrm{Hg}$ in the right ventricle (Table 1), a systolic pressure of $51 \mathrm{~mm} \mathrm{Hg}$ in the main pulmonary artery, and a wedge pressure of 20 $\mathrm{mm} \mathrm{Hg}$ in the right pulmonary artery. Magnetic resonance imaging showed no increase in pericardial thickness. These findings were consistent with RCM.
Table 1 Hemodynamic Data of Case 1

\begin{tabular}{lllc}
\hline \multicolumn{1}{c}{ position } & pressure & position & pressure \\
\hline SVC & $11 / 6(6)$ & RV outflow & 50/EDP10 \\
RA mid & $11 / 6(6)$ & RV inflow & $52 /$ EDP10 \\
IVC & $11 /(6)$ & LV & $97 /$ EDP28 \\
rt-PA branch & $48 / 18(31)$ & AsAo & $96 / 41 /(64)$ \\
rt-PA wedge & $28 / 22(20)$ & & \\
It-PA branch & $52 / 20(32)$ & & \\
m-PA & $51 / 18(32)$ & & \\
\hline
\end{tabular}

SVC: superior vena cava, IVC: inferior vena cava, RA: right atrium, PA: pulmonary artery, RV: right ventricle, LV: left ventricle, AsAo: ascending aorta EDP: end-diastolic pressure. Numbers in brackets indicate mean pressure

The patient was initially treated with an intravenous catecholamine (dobutamine), and then with phosphodiesterase III inhibitors. Consequently, she underwent heart transplantation in the United States at the age of 4 years.

\section{Case 2}

A boy was referred to our hospital at 6 years of age because of cardiomegaly and an abnormal electrocardiogram, detected in a school-based heart disease screening. Chest radiography showed a $54.5 \%$ cardiothoracic ratio. Electrocardiography revealed left atrial enlargement and left ventricular hypertrophy (Fig. 2A). Echocardiography showed enlargement of the left atrium and mild left ventricular dysfunction. A diagnosis of dilated cardiomyopathy was made at that time, and the 
Table 2 Hemodynamic Data of Case 2

\begin{tabular}{lllc}
\hline \multicolumn{1}{c}{ position } & pressure & \multicolumn{1}{c}{ position } & pressure \\
\hline SVC & $11 / 8(9)$ & RV outflow & $41 /$ EDP10 \\
RA mid & $11 / 7(9)$ & RV inflow & $42 /$ EDP11 \\
IVC & $11 / 8(9)$ & LV & $93 /$ EDP22 \\
rt-PA branch & $40 / 20(27)$ & AsAo & $94 / 62 /(76)$ \\
rt-PA wedge & $26 / 24(22)$ & & \\
It-PA branch & $41 / 21(27)$ & & \\
m-PA & $41 / 20(26)$ & & \\
\hline
\end{tabular}

Abbreviations see in Table 1

patient visited our hospital once a year thereafter.

At the age of 10 years 10 months, the boy's heart dysfunction rapidly worsened. The left ventricular ejection fraction decreased from $53 \%$ to $46 \%$. The plasma BNP level increased from 712 to $1,280 \mathrm{pg} /$ $\mathrm{mL}$ (Fig. 3). Because the patient complained of occasional chest pain, he was referred to our hospital for cardiac catheterization. Chest radiography showed cardiomegaly (cardiothoracic ratio, 54\%), with a double silhouette along the right cardiac border indicating left atrial enlargement (Fig. 4). Electrocardiography revealed sinus rhythm, left atrial enlargement, left- and right-ventricular hypertrophy, and ST-T depression (Fig. 2B). Twenty-four-hour electrocardiography showed no significant arrhythmia. Echocardiography showed an anatomically normal heart with left atrial dilatation (left atrial/aortic ratio, 1.70), no tricuspid valve regurgitation, and mild left ventricular dysfunction (ejection fraction, $46 \%$ ). The left ventricular enddiastolic dimension was $48 \mathrm{~mm}$ (reference range: 24$42 \mathrm{~mm}$ ), and the left ventricular end-diastolic wall thickness was $7.3 \mathrm{~mm}$ (reference range: $5-7 \mathrm{~mm}$ ). The early diastolic mitral annular velocity (e') recorded with tissue Doppler echocardiography was $3.78 \mathrm{~cm} / \mathrm{sec}$. The hemodynamic measurements revealed an elevated end-diastolic pressure of $22 \mathrm{~mm}$ $\mathrm{Hg}$ in the left ventricle and $10 \mathrm{~mm} \mathrm{Hg}$ in the right ventricle, along with a systolic pressure of $41 \mathrm{~mm}$ $\mathrm{Hg}$ in the main pulmonary artery and wedge pressure of $22 \mathrm{~mm} \mathrm{Hg}$ of right pulmonary artery (Table 2). Mild mitral valve regurgitation was revealed by left ventriculography. A diagnosis of RCM was made. The patient was treated with diuretics, $\beta$-blocking agents, angiotensin-converting enzyme inhibitors, and digoxin. Two months later, he died suddenly prior to receiving a heart transplant.

\section{Discussion}

Because RCM and constrictive pericarditis share similar clinical and hemodynamic features, including altered ventricular diastolic filling, it is often difficult to distinguish these two conditions by means of clinical examination and conventional testing. Recently, it has been reported that in adults BNP levels are significantly higher in RCM $(825.8 \pm 172.2$ $\mathrm{pg} / \mathrm{mL})$ than in constrictive pericarditis $(128.0 \pm 52.7$ $\mathrm{pg} / \mathrm{mL})^{5}$. Therefore, the plasma BNP level might be used as a tool for differential diagnosis. In our 2 cases in children, the plasma levels of BNP were higher than $500 \mathrm{pg} / \mathrm{mL}$, consistent with the previous report on cases in adults. Moreover, the plasma levels of BNP paralleled the clinical condition. In both of our patients, the BNP levels were around $700 \mathrm{pg} / \mathrm{mL}$ when their conditions were stable but increased markedly (to 1,290 and 1,280 $\mathrm{pg} / \mathrm{mL}$, respectively) when the patients' conditions deteriorated. Thus, monitoring the plasma BNP level might be useful not only for diagnosis but also for evaluation of disease status in children with RCM.

The early diastolic mitral annular velocity (e') recorded with tissue Doppler echocardiography has been reported to accurately discriminate between $\mathrm{RCM}$ and constrictive pericarditis $\mathrm{s}^{6-9}$. In the second of our 2 patients, e' decreased to $3.78 \mathrm{~cm} / \mathrm{sec}$ (normal range: $8.0-16.0 \mathrm{~cm} / \mathrm{sec}$ ), indicating altered ventricular relaxation due to primary myocardial disease. The combination of plasma BNP and e' measured with tissue Doppler echocardiography could allow the more precise, noninvasive diagnosis of RCM.

The clinical course after a diagnosis of RCM in childhood has been reported to be an accelerated deterioration in comparison with that seen in adults $^{3.4}$. Medical treatment of these patients has not been effective over time, and only heart transplantation seems a reliable treatment. However, it is difficult to decide the timing of heart transplantation because of the variability of each individual's natural history. Moreover, no consistent 
predictors for progression of $\mathrm{RCM}$ have yet been identified. According to one report, RCM patients at risk of sudden death often have symptoms such as chest pain or syncope without ongoing heart failure $^{10}$. The authors conclude that these symptoms are caused by ischemia and suggest immediate evaluation including electrocardiography. However it has been reported by others that ST depression in RCM patients with abnormal ST-T segments at rest is not necessarily related to myocardial ischemia, but is rather characteristics of $\mathrm{RCM}^{11}$. So far there is no simple reliable method for evaluating myocardial ischemia in pediatric RCM patients. We recommend that these patients be followed up more carefully using not only conventional examinations but also new methods, such as plasma BNP levels and tissue Doppler echocardiography. We expect that the evaluation of clinical state by a suitable combination of these methods will have important implications in improved prognosis of RCM in children.

The young girl in our first case above was listed for heart transplantation just after symptoms of heart failure appeared and was successfully conducted to transplant. The boy in our second case, in retrospect, might have benefitted by being followed and treated more aggressively, perhaps by implanting a cardioverter-defibrillator and by being listed earlier for transplant. These two cases show that careful and frequent monitoring of electrocardiograms, accurate evaluation of myocardial ischemia, suitable management of heart failure, and proper timing of listing for heart transplantation are critical aspects of the management of pediatric RCM. Evidence is mounting that plasma BNP levels and tissue Doppler echocardiography are important tools that may lead to more accurate diagnosis and more effective treatment of RCM in children.

\section{References}

1. Nugent AW, Daubeney PE, Chondros P, et al.: National Australian Childhood Cardiomyopathy Study. N Engl J Med 2003; 348: 1639-1646.

2. Lipshultz SE, Sleeper LA, Towbin JA, et al: The incidence of pediatric cardiomyopathy in two regions of the United States. N Engl J Med 2003; 348: 16471655 .

3. Weller RJ, Weintraub R, Addonizio LJ, Chrisant MR, Gersony WM, Hsu DT: Outcome of idiopathic restrictive cardiomyopathy in children. Am J Cardiol 2002; 90: 501-506.

4. Russo LM, Webber SA: Idiopathic restrictive cardiomyopathy in children. Heart 2005; 91: 11991202.

5. Leya FS, Arab D, Joyal D, et al:: The efficacy of brain natriuretic peptide levels in differentiating constrictive pericarditis from restrictive cardiomyopathy. J Am Coll Cardiol 2005; 7; 45: 19001902.

6. Rajagopalan N, Garcia MJ, Rodriguez L, et al.: Comparison of new Doppler echocardiographic methods to differentiate constrictive pericardial heart disease and restrictive cardiomyopathy. Am J Cardiol 2001; 87: 86-94.

7. Ha JW, Ommen SR, Tajik AJ, et al.: Differentiation of constrictive pericarditis from restrictive cardiomyopathy using mitral annular velocity by tissue Doppler echocardiography. Am J Cardiol 2004; 94: 316-319.

8. McCall R, Stoodley PW, Richards DA, Thomas L: Restrictive cardiomyopathy versus constrictive pericarditis: making the distinction using tissue Doppler imaging. Eur J Echocardiogr 2008; 9: 591594.

9. Sengupta PP, Krishnamoorthy VK, Abhayaratna WP, et al: Comparison of usefulness of tissue Doppler imaging versus brain natriuretic peptide for differentiation of constrictive pericardial disease from restrictive cardiomyopathy. Am J Cardiol 2008; 102: 357-362.

10. Rivenes SM, Kearney DL, Smith EO, Towbin JA, Denfield SW: Sudden death and cardiovascular collapse in children with restrictive cardiomyopathy. Circulation 2000; 102: 876-882

11. Hayashi T, Tsuda E, Kurosaki K, Ueda H, Yamada O, Echigo S: Electrocardiographic and clinical characteristics of idiopathic restrictive cardiomyopathy in children. Circ J 2007; 71: 15341539 .

(Received, June 17, 2009)

(Accepted, September 14, 2009) 